\title{
Multiple helical modes of vortex breakdown
}

\section{Sørensen, Jens Nørkær; Naumov, I. V.; Okulov, Valery}

Published in:

Journal of Fluid Mechanics

Link to article, DOI:

10.1017/jfm.2011.308

Publication date:

2011

\section{Document Version}

Publisher's PDF, also known as Version of record

Link back to DTU Orbit

\section{Citation (APA):}

Sørensen, J. N., Naumov, I. V., \& Okulov, V. (2011). Multiple helical modes of vortex breakdown. Journal of Fluid Mechanics, 683, 430-441. https://doi.org/10.1017/jfm.2011.308

\section{General rights}

Copyright and moral rights for the publications made accessible in the public portal are retained by the authors and/or other copyright owners and it is a condition of accessing publications that users recognise and abide by the legal requirements associated with these rights.

- Users may download and print one copy of any publication from the public portal for the purpose of private study or research.

- You may not further distribute the material or use it for any profit-making activity or commercial gain

- You may freely distribute the URL identifying the publication in the public portal

If you believe that this document breaches copyright please contact us providing details, and we will remove access to the work immediately and investigate your claim. 


\title{
Multiple helical modes of vortex breakdown
}

\author{
J. N. Sørensen ${ }^{1} \dagger$, I. V. Naumov ${ }^{2}$ and V. L. Okulov ${ }^{1}$ \\ ${ }^{1}$ Department of Mechanical Engineering and Center for Fluid Dynamics, \\ Technical University of Denmark, Nils Koppels Allé, 403, DK-2800 Lyngby, Denmark \\ 2 Institute of Thermophysics, SB RAS and Novosibirsk State University, 630090, Novosibirsk, Russia
}

(Received 21 January 2011; revised 20 April 2011; accepted 11 July 2011;

first published online 19 August 2011)

Experimental observations of vortex breakdown in a rotating lid-driven cavity are presented. The results show that vortex breakdown for cavities with high aspect ratios is associated with the appearance of stable helical vortex multiplets. By using results from stability theory generalizing Kelvin's problem on vortex polygon stability, and systematically exploring the cavity flow, we succeeded in identifying two new stable vortex breakdown states consisting of triple and quadruple helical multiplets.

Key words: vortex breakdown, vortex instability

\section{Introduction}

Vortex breakdown is a phenomenon that appears in many practical problems in aerodynamics, geophysics and engineering sciences. For example, it is observed in tip vortices behind wings and propellers, in atmospheric tornadoes and cyclones, and in flame-holders in combustion devices. Vortex breakdown is characterized by a structural change of the vortex core, associated with an abrupt deceleration of the axial velocity on the vortex axis, which sometimes develops into a recirculation zone. The two predominant breakdown configurations, namely the bubble and the spiral, were first identified by Lambourne \& Bryer (1961) over delta wings. Later, in a pipe flow, Sarpkaya (1971) observed a third type: the double helix. Although now seven different states of vortex breakdown have been identified (Faler \& Leibovich 1978; Sarpkaya 1995; Khoo et al. 1997), only one of them, the double helix, contains stable multiple modes.

A simple experimental means to study the appearance of vortex breakdown is to create a swirling flow by rotating an end-wall in a cylindrical enclosure (see figure 1). In this experiment the rotating lid forces the fluid to perform a rotating motion around the centre axis. Due to centrifugal forces the fluid close to the lid moves away from the centre, causing as a result a meridional circulation. The fluid then travels downwards along the outer wall and turns inwards near the fixed bottom. Close to the centre axis it rises vertically, forms a concentrated vortex structure due to the conservation of angular momentum, and returns to the rotating lid. Changes of the flow structures depend on two parameters: the aspect ratio of container height to disk radius $H / R_{D}$, and the Reynolds number, $R e=\Omega\left(R_{D}\right)^{2} / \nu$, where $\Omega$ is the angular velocity of the disk and $v$ is the kinematic viscosity of the working fluid. Using this

$†$ Email address for correspondence: jns@mek.dtu.dk 
(a)

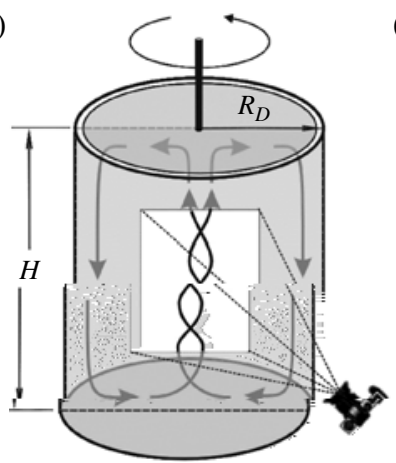

(b)

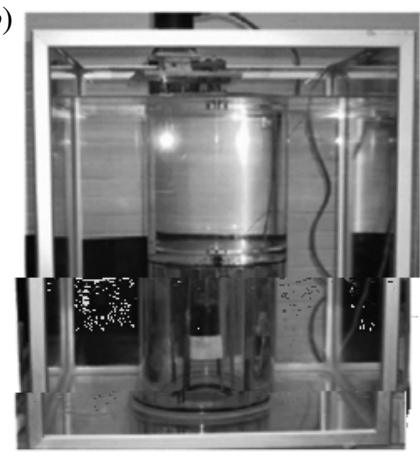

$(c)$

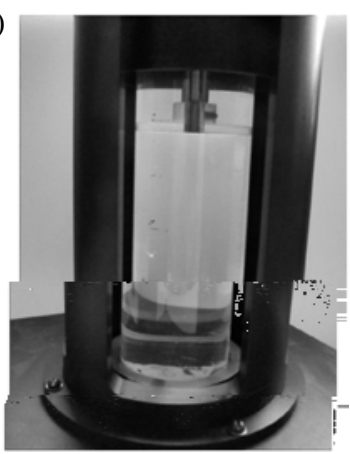

FIGURE 1. Experimental set-ups: (a) sketch; photos of the set-ups at (b) DTU and (c) IT.

configuration, Escudier (1984) observed bubble breakdown with multiple recirculation zones, consisting of up to three axisymmetric recirculating breakdown structures. He mapped the various regions on a Reynolds number versus aspect ratio diagram in which he also depicted the boundary at which the flow becomes unsteady. Escudier, however, limited the experiments to aspect ratios between 1.0 and 3.5. Later studies, by e.g. Lopez (1990, 2006) and Sørensen, Naumov \& Mikkelsen (2006), confirmed and extended the observations of Escudier, but did not detect new breakdown states. In a recent study we focused the investigation on higher aspect ratios, with $H / R_{D}$ going from 3.3 to 5.5, and compared the observed critical Reynolds numbers to values obtained from numerical simulations (Sørensen et al. 2009).

In the present study two new experimental set-ups have been designed, which allow us in a systematic manner to detect multiple helix modes, or vortex multiplets, associated with vortex breakdown in swirl flows. We base this search on the results from a stability analysis (Okulov 2004; Okulov \& Sørensen 2007) showing that an assigned vortex flow can significantly enhance the stability of vortex multiplets. First, a full theoretical analysis of the effect of an intensively swirling assigned flow is performed to determine the stability properties of multiplets. Next, the results of this analysis have been used experimentally to study the various flow regimes appearing in a closed cylindrical cavity with a rotating lid. As a result, we have detected new flow regimes with pronounced stable multiple helical modes of vortex breakdown.

\section{Theoretical background}

There have been many attempts to discover new states of vortex breakdown, including regimes with multiple modes. For example, in a swirling jet, a triple mode was identified to appear just before the onset of vortex breakdown (Billant, Chomaz \& Huerre 1998). However, in most cases a complex and unsteady regime prevails which involves a competition between double and triple helical modes, which makes it extremely difficult to identify and study multiple vortex modes. Hence, a prerequisite for detecting stable breakdown configurations is to look for possible equilibrium states of vortex systems. A simplification of the stability problem is to study the steady rotation of an $\mathrm{N}$-gon of point vortices (or rectilinear vortex filaments). The first investigation of steady point vortex configurations dates back to the work of Lord Kelvin (1878) written partially in view of his vortex atom theory. Later Thomson (1883) won the Adams prize for a proof on the existence of linear stability of an array of polygonal point vortices for $N<7$. A complete analysis of the stability for point 


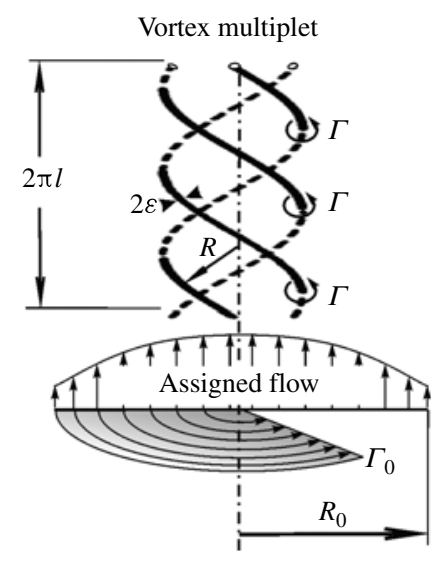

FIGURE 2. Sketch of a vortex multiplet embedded in an assigned flow.

vortices was later carried out by Havelock (1931), who determined the well-known stability condition as

$$
s(N-s)-2(N-1)<0
$$

for any $s$, where $s \in[0, N-1]$ is the sub-harmonic wavenumber of the vortex polygon disturbances.

The task of finding regular equilibrium structures consisting of identical vortices in real fluids (including the search for multiple modes associated with vortex breakdown) encounters considerable difficulties, and this problem is still far from being exhaustively studied. The difficulties are related to various factors, primarily to the fact that rectilinear vortex configurations in real liquids take various spiral forms, of which the most frequently encountered is the helical vortex. Helical vortices can form different equilibrium systems, consisting of identical cores with axes lying on a common generating cylinder in the form of doublets, triplets or multiplets. Such vortex structures represent helical counterparts to point vortex polygons, which, in the limit as the helical pitch tends to infinity, completely coincide with these polygons. However, the conditions for existence of stable helical vortex multiplets depend on the helical pitch and are thus more stringent than for point vortices. Recently it was shown (Okulov 2004) that stable vortex multiplets comprising up to seven vortices may exist for sufficiently large values of the dimensionless torsion of the helix axis (defined as the ratio between the helical pitch $2 \pi l$ and the generating cylinder circuit $2 \pi R$, in accordance with the notation shown in figure 2). As the number of vortices in the multiplet increases, the critical torsion value (under which the multiplet becomes unstable) increases, and multiplets consisting of seven or more helical vortices become unconditionally unstable for any finite value of the torsion. The instability makes it a very difficult task to detect and study multiple helix modes during vortex breakdown. We base our search on the results from the stability analysis by Okulov \& Sørensen (2007) showing that the ambient swirl flow can significantly enhance the stability of vortex multiplets.

As basis for the stability analysis, an assigned swirl flow (figure 2) is represented by an analytical solution describing a columnar helical vortex having the same helical pitch, $2 \pi l$, as the vortex multiplets, and a Gaussian distribution of the axial vorticity component. In a cylindrical coordinate system $(r, \theta, z)$, this results in the following 
expression:

$$
\omega_{z}=\frac{\Gamma_{0}}{\pi R_{0}^{2}} \exp \left(-\frac{r^{2}}{R_{0}^{2}}\right), \quad \omega_{\theta}=r \omega_{z} / l \quad \text { and } \quad \omega_{r}=0 .
$$

This generates the following azimuthal $\left(w_{\theta}\right)$ and axial $\left(w_{z}\right)$ velocity components:

$$
\left.\begin{array}{l}
w_{\theta}=\frac{\Gamma_{0}}{2 \pi r}\left[1-\exp \left(-\frac{r^{2}}{R_{0}^{2}}\right)\right], \\
\left.w_{z}=w_{0}-\frac{r}{l} w_{\theta} \quad \text { or } \quad w_{z}=w_{0}-\frac{\Gamma_{0}}{2 \pi l}\left[1-\exp \left(-\frac{r^{2}}{R_{0}^{2}}\right)\right] \cdot\right\}
\end{array}\right\}
$$

This class of one-dimensional solutions (2.3) describes a columnar vortex structure with a dense distribution of helical vortex lines of pitch $2 \pi l$, effective vortex core radius $R_{0}$, and a total circulation $\Gamma_{0}$ (Kuibin \& Okulov, 1996; or Alekseenko et al. 1999, paragraph 4.1). Note that the same vortex model (2.3), in the sense of Batchelor's vortex (Batchelor 1964) or the $q$-vortex, is commonly used to describe experimental swirl flows (Faler \& Leibovich 1978; Escudier 1988; Alekseenko et al. 1999) and in instability studies of swirling flows (see Heaton \& Peake 2007 and references therein).

We have analysed the linear stability problem of small displacements from an equilibrium state for a multiplet of $N$ helical vortices embedded in the assigned flow defined by (2.3). The vortex axes in this multiplet are placed on a cylindrical generator of radius $R$ with an angular shift of $2 \pi / N$ (figure 2). Each vortex is determined by an identical core with dimensionless radius $\sigma=\varepsilon / R$, dimensionless pitch $\tau=2 \pi l / R$, and circulation $\Gamma$. The stability analysis (Okulov 2004; Okulov \& Sørensen 2007) leads to the following stability condition:

$$
s(N-s) A-2(N-1)+4 N \gamma B+C<0,
$$

where $A=(t / \tau)^{3}, B=\left[(t / \delta \tau)^{2}+1\right] \exp \left(-\delta^{-2}\right)-1$ and

$$
\begin{aligned}
C= & 2 \frac{N-2}{\tau^{2}}+\frac{1+2\left(\tau^{2}-\tau t\right)}{\tau t}+\frac{\tau^{3}}{t^{9}}\left(2 \tau^{4}-6 \tau^{2}+\frac{3}{4}\right) \frac{\zeta(3)}{N^{2}} \\
& +\frac{\tau^{2}-\frac{1}{4}}{\tau t^{3}}\left[E+\psi\left(-\frac{s}{N}\right)-\frac{N}{s}+\frac{3}{4}-2 \tau^{2}-\ln \left(N \sigma \frac{t^{3}}{\tau}\right)\right] .
\end{aligned}
$$

The parameters $A$ and $C$ result from the helical form of the vortex multiplet, where $t=\left(1+\tau^{2}\right)^{1 / 2}, E=0.577215 \ldots$ is the Euler constant, $\zeta(3)=1.20206 \ldots$ is the Riemann zeta function, and the psi function $\psi(\cdot)$ takes values such as $\psi(-1 / 2)=0.0395 \ldots, \psi(-1 / 3)=1.6818 \ldots, \psi(-2 / 3)=-1.6320 \ldots, \psi(-1 / 4)=$ $2.9141 \ldots, \psi(-3 / 4)=-2.8941 \ldots$

The coupling between the assigned flow and the flow induced by the vortex multiplet is characterized by the dimensionless parameters $\gamma=\Gamma_{0} / N \Gamma$ (the circulation ratio) and $\delta=R_{0} / R$ (the ratio of the characteristic radial dimensions). In the limit as $l \rightarrow \infty$ (whereby the vortices become rectilinear, corresponding to point vortices) and without assigned flow, i.e. $\gamma=0$, the stability condition for helical vortices, (2.4), degenerates to (2.1).

The third term in (2.4) describes the effect of the assigned flow on the stability of the helical multiplets and shows that the vortex configuration in most cases becomes more stable when $\gamma$ increases. Figure 3 shows the neutral curves corresponding to 

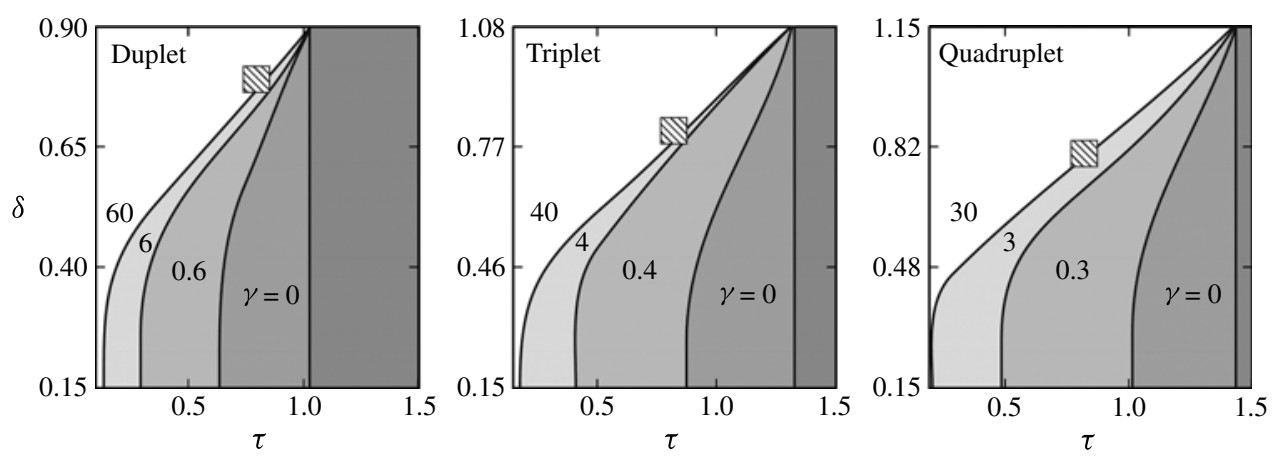

FIGURE 3. Neutral curves showing the stability of a helical vortex duplet, triplet and quadruplet, respectively, in an assigned swirl flow, plotted as functions of dimensionless vortex pitch $\tau$ and ratio $\delta$ of the vortex size of the assigned flow and the multiplets for various values of the circulation ratio $\gamma$. The side of a neutral curve with a more intense colour corresponds to a stable flow regime.

the stability condition (2.4) for duplets, triplets and quadruplets of helical vortices, plotted as a function of the dimensionless vortex pitch $\tau$ and the ratio $\delta$ of the characteristic radial dimensions. Different curves correspond to increasing values of the relative circulation parameter: $N \gamma=0,1.2,12$ and 120. The side of a neutral curve with a more intense colour corresponds to a stable flow regime. As seen, the stable flow regimes expand when the strength of the assigned flow increases. Thus, the presence of the assigned flow stabilizes the vortex multiplets. The diagrams constitute an important diagnostics tool for detecting systematically vortex multiplets.

\section{Experimental set-up}

Taking the stability analysis into account, we have attempted to detect stable multiple helix modes of vortex breakdown in two different experimental set-ups consisting of a swirling flow in a closed cylindrical cavity with a rotating lid, where a central concentrated vortex structure is superposed on a strong vortex flow (see the sketch and photos in figure 1). The experiments were carried out as a collaboration between the Technical University of Denmark (DTU) and RAS Institute of Thermophysics (IT) in Novosibirsk. The experiments at DTU were carried out in a cylindrical Plexiglas container with an inner diameter of $288 \mathrm{~mm}$. The whole cylindrical container is placed inside a rectangular box of dimensions $700 \mathrm{~mm} \times 700 \mathrm{~mm} \times 800 \mathrm{~mm}$ that is made of glass and filled with tap water, in order to minimize optical aberrations and thermal changes. An $80 \%$ glycerin-water mixture was used as working fluid. The top lid is connected to two stepping motors that allow the lid to be rotated continuously and lifted automatically in order to vary the height of the flow domain. The experiments at IT were carried out in a cylindrical container of quartz glass, with an inner diameter of $56 \mathrm{~mm}$, which was filled with tap water. The top lid is rotated by a stepping motor. In both investigations, the aspect ratio $H / R_{D}$ is varied in the range from 1.0 to 6.0 with steps of 0.1 and Reynolds numbers ranged from 2000 to 3500 with a step of 100. The two experimental set-ups are shown in figure 1. The accuracy of both set-ups is estimated as follows: the error in the definition of the aspect ratio is less than $0.15 \%$; the variation in angular velocity during a revolution is less than $0.05 \%$; and the total error of $R e$ does not exceed \pm 10 in a range of Reynolds numbers from 2000 to 5000. 


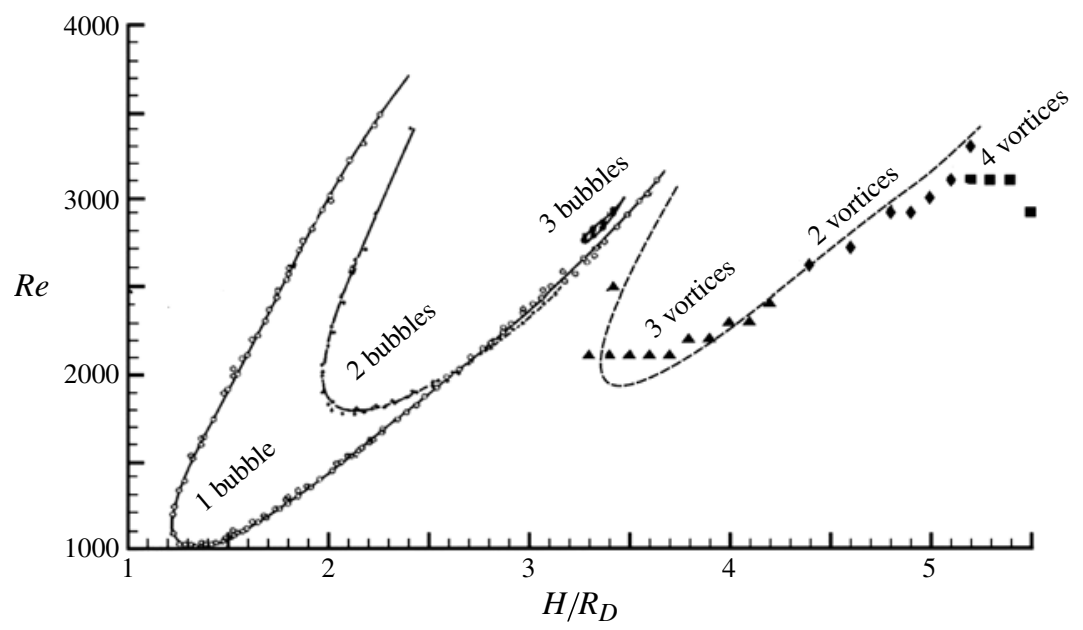

FIGURE 4. Map of critical Reynolds number for different modes of the vortex breakdown as a function of the cylinder aspect ratio: solid line and small symbols correspond to onset of bubble modes (Escudier 1984); dashed line and large symbols indicate the appearance of multiple modes (present study).

\section{Results}

Most studies of the flow in a rotating cavity have been carried out for aspect ratios in the range $1 \leqslant H / R_{D} \leqslant 3.5$. In our laboratories a series of experiments (Naumov et al. 2003; Okulov, Sørensen \& Varlamova 2004; Sørensen et al. 2006) have been performed aimed at verifying experimental techniques by comparison with flow visualizations. For aspect ratio $1 \leqslant H / R_{D} \leqslant 3.2$ only bubble modes of vortex breakdown with one, two or three steady axisymmetric recirculating zones appeared on the cylinder axis. On increasing further the aspect ratio from 3.3 to 6.0 the flow is subject to a complicated transition process in which it becomes unsteady and three-dimensional without generating steady-state bubble breakdown. To study in detail the flow behaviour we apply a measurement technique that combines particle image velocimetry (PIV) with laser Doppler anemometry (LDA) (see Naumov et al. 2003; Sørensen et al. 2006). First, LDA is employed to determine the critical Reynolds numbers at which the basic steady and axisymmetric flow loses its stability. Next, a detailed analysis of the spatial structures in a horizontal cross-section is performed using phase-averaged PIV images. The flow field, which is taken in a horizontal crosssection located one-quarter of a cylinder height from the bottom, is viewed through the transparent flat bottom of the container.

An overview of the obtained results can be seen in figure 4, which depicts boundaries of critical Reynolds numbers at which vortex breakdown appears. The left-hand part, which shows the critical Reynolds numbers as a function of aspect ratios in the interval from 1.0 to 3.8, is taken from the experiments of Escudier (1984). The regions referred to as 1,2 and 3 bubbles denote the different types of bubble behaviour observed by Escudier. For $1 \leqslant H / R_{D} \leqslant 3.3$ the onset of vortex breakdown is associated with axisymmetric flow behaviour after which the flow first becomes threedimensional at higher Reynolds numbers in the unsteady regime. For $H / R_{D}>3.3$, however, our experiments show that transition to vortex breakdown takes place directly from a steady axisymmetric flow state to a full three-dimensional and unsteady flow state. This leaves us with the interesting question about what happens exactly at the 

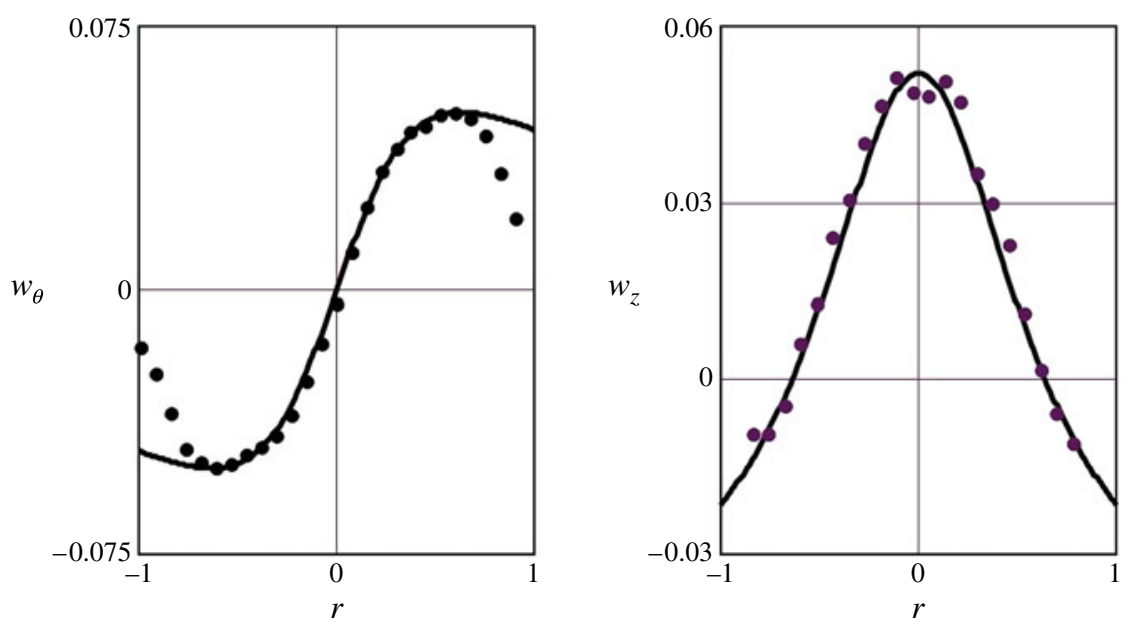

FIGURE 5. A comparison between measured average azimuthal $\left(w_{\theta}\right)$ and axial $\left(w_{z}\right)$ velocity profiles (points) and analytical solutions (solid lines) described by (2.3) for flow regimes with $H / R_{D}=2, R e=1500$.

critical Reynolds number, i.e. what kind of three-dimensional structure is associated with the transition. Using the aforementioned experimental technique we found that the structures are associated with the appearance of helical multiplets consisting of up to four vortices. This is mapped in figure 4 , where a helical triplet is seen to govern the steady-unsteady transition in the range $3.2 \leqslant H / R_{D} \leqslant 4.4$, whereas the steady/unsteady transition in the range $4.5 \leqslant H / R_{D} \leqslant 5.2$ is governed by a helical dipole and in the range $5.3 \leqslant H / R_{D} \leqslant 6.0$ it is governed by the appearance of a helical quadruplet.

To compare the results to the stability analysis described by Okulov (2004) and Okulov \& Sørensen (2007) we analyse the PIV-processed flow field using (2.2)-(2.4). To illustrate this procedure, consider two steady flow fields at two different positions in the parameter range, which are both close to a critical Reynolds number. The first is located in the stable axisymmetric flow regime at $H / R_{D}=2$ and $R e=1500$ (figure 5). From the centre of the cavity and 0.8 radii outward, where the influence of the wall can be neglected, the measured tangential and axial velocity profiles are compared to velocity profiles determined from (2.3). A good agreement is found between the measured distribution and the analytical one using the parameters $R_{0}=0.63 R_{D}, \Gamma_{0}=0.4, w_{0}=0.052$, and $l=0.63 R_{D}$, which describes a swirling jet-flow with a Gaussian vorticity distribution. This illustrates that, in the axisymmetric flow domain, the bulk flow in the inner part of the cylinder is governed by a simple Gaussian vortex. The axisymmetric picture changes markedly when aspect ratio and Reynolds number are increased. Figure 6 shows a comparison at $H / R_{D}=3.5$ and $R e=2300$ between an experimentally obtained phase-averaged tangential velocity profile and a tangential velocity profile obtained from (2.3) using the values $R_{0}=0.45 R_{D}$, and $\Gamma_{0}=0.24$. Here good agreement only exists in the right part of the plot, whereas the left part of the experimental profile deviates markedly from the axisymmetric solution. To determine the structure of the perturbed flow field, we subtract the axisymmetric solution given by (2.3) from a phase-averaged velocity field obtained from 20 phase-averaged PIV-samples using the LDA-PIV diagnostics technique described above. The result of this is shown as the zoomed part in figure 6. The subtracted profile of the tangential velocity around the vortex core 

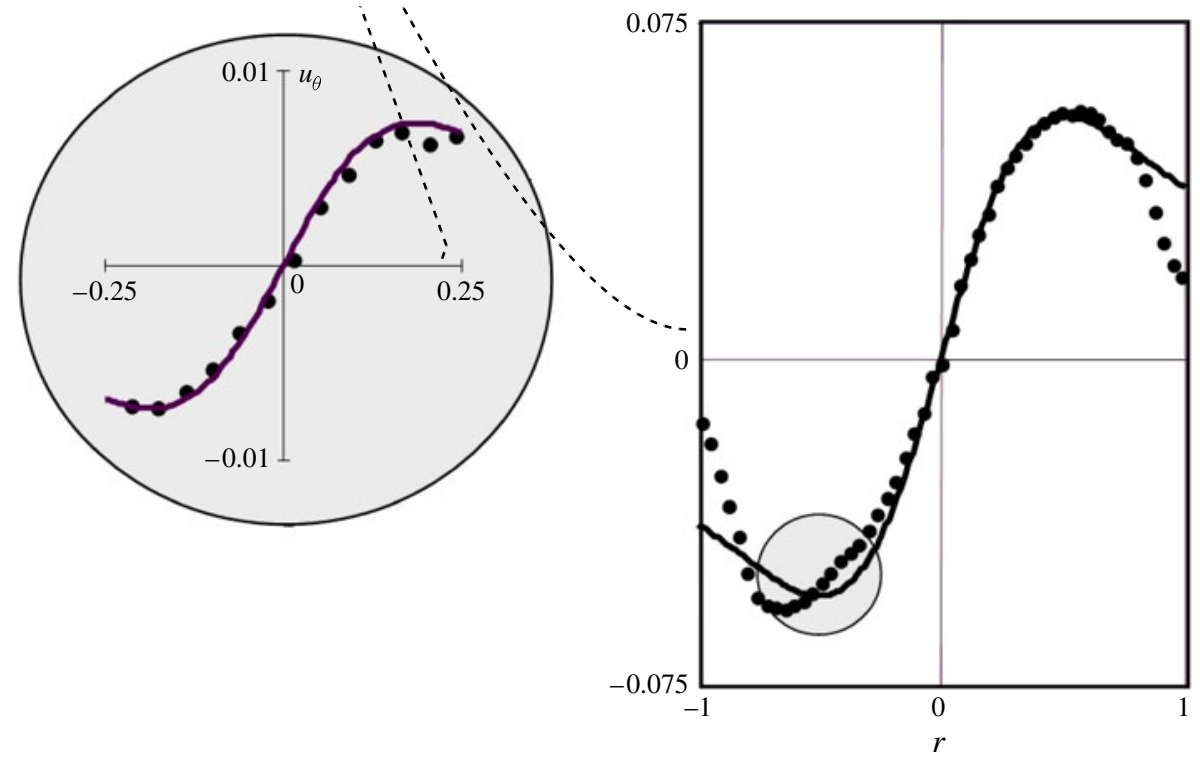

FIGURE 6. Instantaneous azimuthal velocity profile (points), measured along the horizontal diameter of the triplet shown in figures $8(a)$ and $9(a)$, and a fitted curve from the analytical model (2.3) (solid lines) for a flow regime with $H / R_{D}=3.5, R e=2300$. On the zoomed part of the plot the extracted azimuthal velocity, denoted $u_{\theta}$, appearing around a vortex in the triplet has been fitted by the analytical model (2.3).

FIGURE 7. Rotation of triplet shown at three different instants $\left(R e=2300 ; H / R_{D}=3.5\right)$.

of the multiplets was again compared to the model (2.3) for which a good agreement was found with an effective core size $\varepsilon=0.37 R_{0}$, circulation $\Gamma=0.05 \Gamma_{0}(\gamma=6.6)$ and a vortex centre placed at radius $R=0.55 R_{D}(\delta=0.8)$ from the centre of the cavity. The multiple structure of these vortices is demonstrated in figure 7 where plots of the disturbed velocity at three different times reveal the existence of a flow structure with three equidistantly located vortices. The images of the velocity are obtained by statistical averaging of 20 selected PIV-samples and subtracting from this the basic axisymmetric vortex (2.3). As a result these plots show the appearance of a 'triplet symmetry' and demonstrate that the flow instability is associated with a uniform rotation of a vortex triplet embedded in strong assigned axisymmetric flow.

Figure 8 shows PIV-determined isolines of axial vorticity for three different flow patterns. The colours of the iso-plot indicate the strength of the vorticity, with the lightest being the strongest. The top plots show vorticity values as they appear directly 
(a)

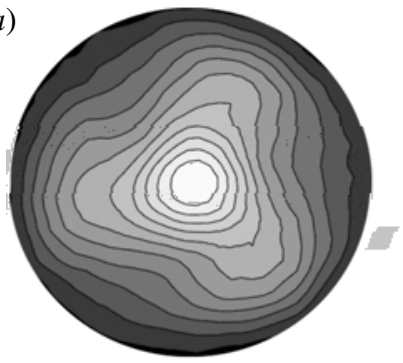

(b)

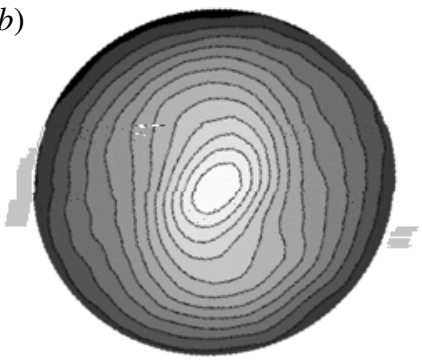

(c)

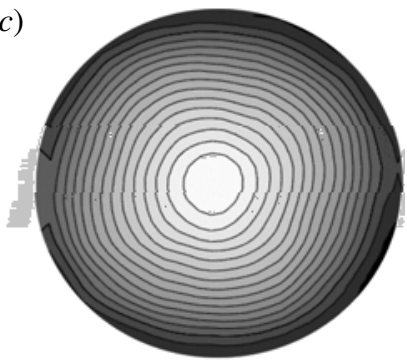

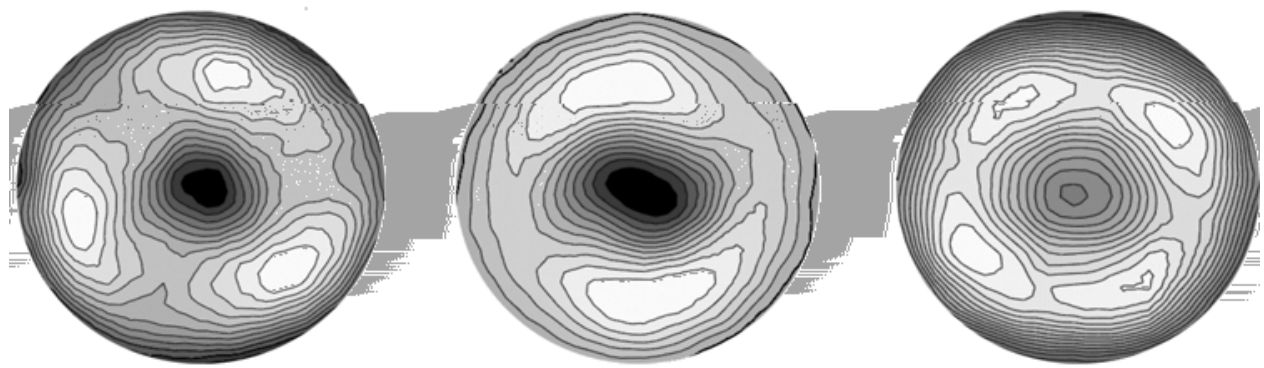

FIGURE 8. Examples of LDA-PIV diagnostics in a horizontal cross-section showing multiple vortex breakdown: $(a)$ triplet $\left(R e=2300 ; H / R_{D}=3.5\right),(b)$ doublet $(R e=$ $\left.2800 ; H / R_{D}=4.6\right)$, (c) quadruplet $\left(R e=3100 ; H / R_{D}=5.3\right)$. Top row: iso-lines of the vorticity of the original vorticity field. Bottom row: iso-lines of the vorticity without the assigned flow.

from phase-averaged PIV images, whereas the bottom vorticity plots are derived by subtracting the assigned axisymmetric flow (2.3). Thus, by subtracting the assigned flow field from phase-averaged PIV samples, the multiple modes become visible. The vorticity plots are clearly seen to reveal cross-sections of multiple vortex cores with triplet, duplet and quadruplet structures. Note that the light colour giving the value of the vorticiy in the vortex cores of the multiplets has the same sign as the assigned vorticity component. It should be mentioned that the observed multiplets kept a stable form during all the observation time. Thus, after transients had died out, the structures persisted for more than 300 disk rotations.

It should be noted that the observed vortex multiplets differ from rotating waves, such as those used to analyse stability properties of the cavity flow (Gelfgat, Bar-Yoseph \& Solan 2001; Lopez 2006). The waves exhibit a pronounced multipole structure (dipole, tripole, quadrupole, etc.) with four, six, and eight equidistant vortices with an alternating sign of circulation between adjacent vortices. Such structures, however, represent azimuthal perturbations of the axisymmetric flow field (Sørensen et al. 2006). The vortex structures observed in the present study are comprised of helical vortices with the same sign of circulation, which corresponds to the structure stipulated by the aforementioned Kelvin problem concerning the equilibrium of a vortex polygon.

To validate further the existence of these new regimes of vortex breakdown, two different flow visualization experiments were carried out. In the first small air bubbles $(0.1 \mathrm{~mm}$ diameter) were used as tracers to visualize the flow in the set-up located at DTU. As the local circumferential velocity increases at the cavity centre the pressure 


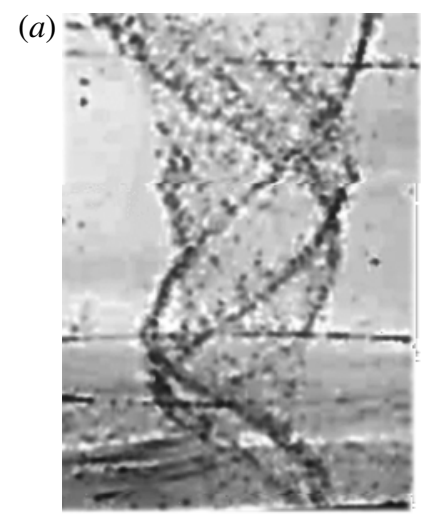

(b)
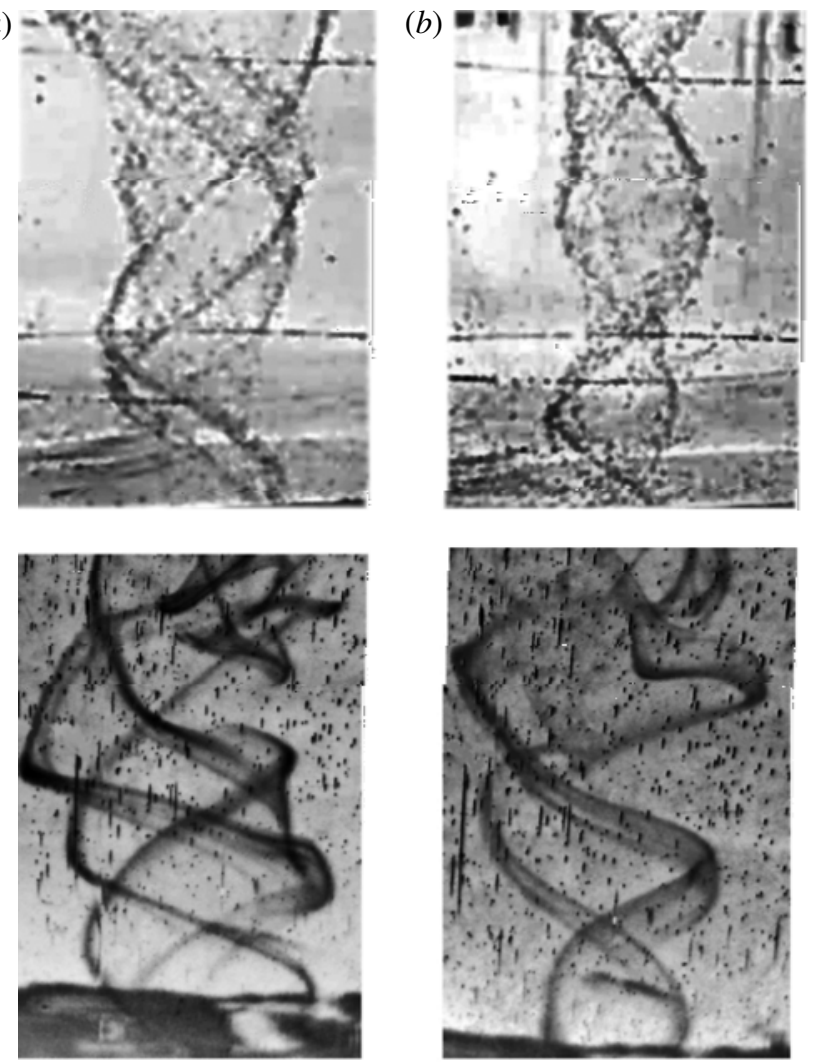

(c)

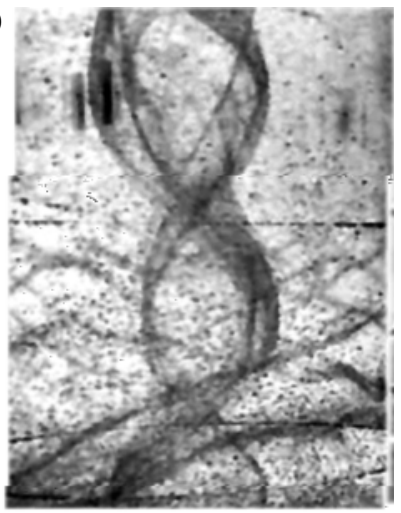

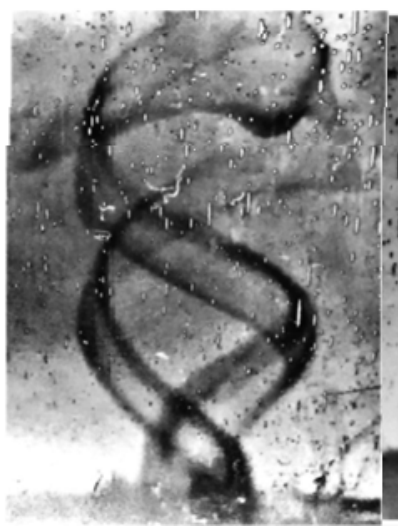

FIGURE 9. Multiple modes of vortex breakdown: $(a)$ triplet $\left(R e=2300 ; H / R_{D}=3.5\right),(b)$ doublet $\left(R e=2800 ; H / R_{D}=4.6\right)$, (c) quadruplet $\left(R e=3100 ; H / R_{D}=5.3\right)$. Top row: bubble visualization (carried out at DTU). Bottom row: dye visualization in (carried out at IT).

decreases and, due to cavitation, forms thin air-filled continuous filaments (see figure 9, top row).

From the air bubble visualizations of the helical vortices it is possible to estimate the dimensionless helical pitch $\tau$ (Alekseenko et al. 1999) which is found to have the same value of approximately $\tau=0.8$, independent of the type of multiplet. By using the difference between the measured tangential velocity profiles and the analytical expression (2.3), it is possible to determine circulation ratios $\gamma$ as well as $\delta$, the ratio between the radius of the assigned flow and the radius of the multiplet. For all the cases in which multiplets were detected these values are found to be in the vicinity of $\gamma \approx 20 / N$ and $\delta \approx 0.8$. We computed the associated $(\tau, \delta, \gamma)$ values and plot them as cross-hatched squares in figure 3 . As seen from the figure, for all three multiplet configurations, i.e. duplet, triplet and quadruplet, the parameters fall on the curve defining the boundary between stable and unstable states of helical vortex multiplets in strong assigned flows. Thus, the appearance of this vortex structure may be explained by the theory proposed above.

The second visualization was made in IT by using dye, which initially was distributed uniformly on the cavity bottom (see figure 9, bottom row). As seen from the figure, both air and dye particles form helical multiplet structures around the cavity centre. As a further validation, the experiments were carried out using both pure water 
(IT) and a $70 \%$ glycerin-water mixture (DTU) as working fluids, showing that the structures did not depend on the actual working fluid used in the experiments. Thus, both the combined LDA-PIV measurements and the two visualization techniques confirm the existence of multiple helical modes of vortex breakdown.

It should be noted that we have only detected stable multiplets with up to 4 vortices. We did observe an unsteady core consisting of 5 vortices, but it was associated with a competition between doublet and quadruplet helical modes and therefore unstable.

\section{Conclusion}

An experimental study has been carried out to detect helical multiplets of vortex breakdown in a rotating cavity flow. The experiments were carried out employing two different experimental set-ups, one located at Institute of Thermophysics in Novosibirsk and the other located at the Technical University of Denmark. In the measurements both a combined LDA-PIV technique and visualizations were performed. The experimental results revealed the existence of vortex breakdown consisting of stable multiplets with up to four helical vortices. The results were in excellent agreement with stability analysis, demonstrating that three-dimensional vortex breakdown is associated with the creation of helical multiplets.

This work was partially supported by grants from the Danish FTP (no. 09-070608), the RFBR (no. 10-08-01093) and a project of the Russian Ministry of Education and Science (no. 2.1.2/12311).

\section{REFERENCES}

Alekseenko, S. V., Kuibin, P. A., Okulov, V. L. \& Shtork, S. I. 1999 Helical vortices in swirl flow. J. Fluid Mech. 382, 195-243.

BAtchelor, G. K. 1964 Axial flow in trailing line vortices. J. Fluid Mech. 20, 645-658.

Billant, P., Chomaz, J. M. \& Huerre, P. 1998 Experimental study of vortex breakdown in swirling jets. J. Fluid Mech. 376, 183-219.

ESCUDIER, M. P. 1984 Observations of the flow produced in a cylindrical container by a rotating endwall. Exp. Fluids 2, 189-196.

Escudier, M. P. 1988 Vortex breakdown: observations and explanations. Prog. Aerosp. Sci. 25, $189-229$.

FALER, J. H. \& Leibovich, S. 1978 An experimental map of the internal structure of a vortex breakdown. J. Fluid Mech. 86 (2), 313-335.

Gelfgat, A. Y., Bar-Yoseph, P. Z. \& Solan, A. 2001 Three-dimensional instability of axisymmetric flow in a rotating lid-cylinder enclosure. J. Fluid Mech. 438, 363-377.

HAVELOCK, T. H. 1931 The stability of motion of rectilinear vortices in ring formation. Phil. Mag. 11, 617-633.

Heaton, C. J. \& Peake, N. 2007 Transient growth in vortices with axial flow. J. Fluid Mech. 587, 271-301.

Khoo, B. C., Yeo, K. S., Lim, D. F. \& He, X. 1997 Vortex breakdown in an unconfined vortical flow. Exp. Therm. Fluid Sci. 14, 131-148.

Kuibin, P. A. \& OKulov, V. L. 1996 One-dimensional solutions for a flow with a helical symmetry. Thermophys. Aeromech. 3, 297-301.

Lambourne, N. C. \& Bryer, D. W. 1961 The bursting of leading-edge vortices. Aero. Res. Counc. $R \& M$ 3282, 1-36.

LopeZ, J. M. 1990 Axisymmetric vortex breakdown. Part 1. Confined swirling flow. J. Fluid Mech. 221, 533-552.

LOPEZ, J. M. 2006 Rotating and modulated rotating waves in transitions of an enclosed swirling flow. J. Fluid Mech. 553, 323-346. 
Naumov, I. V., Okulov, V. L., Mayer, K. E., Sørensen, J. N. \& Shen, W. Z. 2003 LDA-PIV diagnostics and 3D simulation of oscillating swirl flow in a closed cylindrical container. Thermophys. Aeromech. 10 (2), 143-148.

OKulov, V. L. 2004 On the stability of multiple helical vortices. J. Fluid Mech. 521, 319-342.

OKulov, V. L. \& Sørensen, J. N. 2007 Stability of helical tip vortices in a rotor far wake. J. Fluid Mech. 576, 1-25.

Okulov, V. L., Sørensen, J. N. \& Varlamova, E. A. 2004 Emergence of asymmetry and unsteadiness in laboratory simulation of the hydrodynamic structure of a tornado. Izv. Acad. Nauk SSSR Atmos. Ocean. Phys. 40 (2), 169-182.

SARPKAYA, T. 1971 On stationary and travelling vortex breakdowns. J. Fluid Mech. 45 (3), 545-559.

SARPKAYA, T. 1995 Turbulent vortex breakdown. Phys. Fluids 7, 2301-2303.

Sørensen, J. N., Gelfgat, A. Y., Naumov, I. V. \& Mikkelsen, R. 2009 Experimental and numerical results on three-dimensional instabilities in a rotating disk-tall cylinder flow. Phys. Fluids 21 (5), 054102.

Sørensen, J. N., NAumov, I. V. \& Mikkelsen, R. 2006 Experimental investigation in three-dimensional flow instabilities in a rotating lid-driven cavity. Exp. Fluids 41, 425-440.

Thomson, J. J. 1883 A Treatise on the Motion of Vortex Rings. Macmillan.

Thomson, W. (Lord Kelvin) 1878 Floating magnets (illustrating vortex-systems). Nature 18, 13-14. 